\title{
Reaction of Lines of the Rapid Cycling Brassica Collection and Arabidopsis thaliana to Four Pathotypes of Plasmodiophora brassicae
}

Kalpana Sharma, Department of Plant Agriculture, University of Guelph, Guelph, ON, N1G 2W1 Canada and Agriculture and AgriFood Canada, Saskatoon Research Centre, Saskatoon, SK, S7N 0X2 Canada; Bruce D. Gossen, Agriculture and Agri-Food Canada, Saskatoon Research Centre; David Greenshields and Gopalan Selvaraj, Plant Biotechnology Institute, National Research Council of Canada, Saskatoon, SK, S7N 0W9 Canada; Stephen E. Strelkov, Department of Agricultural, Food and Nutritional Science, University of Alberta, Edmonton, AB, T6G 2P5, Canada; and Mary Ruth McDonald, Department of Plant Agriculture, University of Guelph

\begin{abstract}
Sharma, K., Gossen, B. D., Greenshields, D., Selvaraj, G., Strelkov, S. E., and McDonald, M. R. 2013. Reaction of lines of the Rapid Cycling Brassica Collection and Arabidopsis thaliana to four pathotypes of Plasmodiophora brassicae. Plant Dis. 97:720-727.

The clubroot reaction of five Rapid Cycling Brassica Collection (RCBC) lines (Brassica carinata, B. juncea, B. napus, B. oleracea, and B. rapa) and 84 lines of Arabidopsis thaliana to pathotypes 2, 3, 5, and 6 of Plasmodiophora brassicae (as classified on William's system) was assessed. Also, the reaction of the Arabidopsis lines to a single-spore isolate of each of pathotypes 3 and 6 was compared with that of a field isolate. Seedlings were inoculated with resting spores of $P$. brassicae, maintained at 25 and $20^{\circ} \mathrm{C}$ (day and night, respectively), and assessed for clubroot incidence and severity at 6 weeks after inoculation. Several lines of A. thaliana and RCBC exhibited a differential response to pathotype but none of the lines were immune. Among the RCBC lines, $B$. napus was resistant to all of the pathotypes; $B$. oleracea was re-

sistant to pathotypes 2,3 , and $5 ; B$ carinata and $B$. rapa were resistant to pathotypes 2 and 5 ; and $B$. juncea was susceptible to pathotypes 5 and 6 and had an intermediate response to pathotypes 2 and 3. Line Ct1 of A. thaliana was highly resistant to pathotype 2, Pu2-23 was highly resistant to pathotype 5, and Ws-2 and Sorbo were highly resistant to pathotype 6 . These results indicate that the lines of RCBC and A. thaliana have potential for use as model crops for a wide range of studies on clubroot, and could be used to differentiate these four pathotypes of P. brassicae. The reaction of the RCBC lines to pathotype 6 was highly correlated with response under field conditions but the reaction to the single-spore isolates of pathotypes 3 and 6 was not strongly correlated with reaction to the field collections in the Arabidopsis lines.
\end{abstract}

Clubroot, caused by the obligate parasite Plasmodiophora brassicae Woronin, is an economically important disease of crops in the Brassicaceae family. The pathogen induces root malformations (clubs) on infected plants, reducing the capacity for water and nutrient uptake and transport (7). Plants become wilted and stunted, and yield can be reduced when symptoms are severe. Hundreds of millions of long-lived resting spores of $P$. brassicae are produced inside each clubbed root and are released into the soil when the clubs decay and disintegrate (32).

Historically, the impact of clubroot in Canada has been greatest on Brassica vegetables in British Columbia, Ontario, and Quebec (12). However, clubroot has recently become a major concern for canola (Brassica napus L.) producers in the Northern Great Plains, where it has spread rapidly since it was first reported on canola near Edmonton, AB in 2003 (12,13,30). Yield losses in canola can exceed $90 \%(12,13)$. As a result, $P$. brassicae is an emerging threat to the 4.7-million-ha canola industry on the Canadian prairies.

Various host differential sets, consisting of selected Brassica genotypes, have been suggested to differentiate the pathotypes of $P$. brassicae $(5,15,27,33)$. The differential set of Williams is based on the reaction of cabbage ( $B$. oleracea L. var. capitata) 'Jersey Queen' and 'Badger Shipper' and rutabaga (B. napus L. var. napobrassica) 'Laurentian' and 'Wilhelmsburger' (33). The European

Corresponding author: M. R. McDonald, E-mail: mrmcdona@uoguelph.ca

Current address of D. Greenshields: Novozymes BioAg Ltd., Saskatoon, SK, S7R 1A3, Canada

* The $e$-Xtra logo stands for "electronic extra" and indicates that two supplementary tables are included in the online edition.

Accepted for publication 9 December 2012.

http://dx.doi.org/10.1094/PDIS-08-12-0752-RE

(c) 2013 Department of Agriculture and Agri-Food, Government of Canada
Clubroot Differential (ECD) set consists of five genotypes each of B. napus, B. rapa, and B. oleracea (5). These two differential sets have been used to characterize pathogen populations from Canada $(3,22,29)$ and other regions of the world.

Pathotype 6 (ECD 16/02/30) is predominant in the Midwestern United States $(23,33)$ and pathotype 7 (ECD 16/02/31 or ECD $16 / 03 / 31)$ in the eastern United States $(8,33)$. The predominant pathotypes of $P$. brassicae in Canada are 2, 3, 5, and 6, based on the differential set of Williams and confirmed using the ECD set $(28,29)$. Pathotype 6 (equivalent to ECD 16/0/14) is the predominant pathotype in Ontario and British Columbia $(22,29)$. Pathotype 2 (ECD 16/15/31) is present in Quebec $(29,31)$. Pathotype 3 (ECD $16 / 15 / 12$ ) is dominant in Alberta but pathotype 5 (ECD 16/15/0) is also present in that province (29). Pathotype 3 is also the dominant pathotype in Nova Scotia (11). However, the pattern was found to be somewhat different when the pathotypes were assessed using single-spore isolates (35); pathotypes $2,3,6$, and 8 were identified in Alberta; pathotypes 3, 5, and 8 in Ontario; and pathotype 6 in British Columbia. Previous studies indicate that there are potentially more pathotypes that are present at low frequency $(6,12,28)$. Populations of $P$. brassicae in Canada are considered to be less diverse than those in Europe (9) but more diverse than those in the United States $(8,23,33)$.

Arabidopsis thaliana is a host of $P$. brassicae and has been used as a model system for molecular studies on clubroot $(2,14,18,20,26)$. The small, sequenced genome and the availability of many mutant lines of A. thaliana, together with its small stature and short life cycle, make it ideal for use as a model plant in molecular assessments. However, a model crop with a growth habit and architecture more similar to susceptible crop species would be useful for many other kinds of studies. The Rapid Cycling Brassica Collection (RCBC), also known as Wisconsin Fast Plants, consists of Brassica lines that are early flowering, with small stature and a short life cycle, and results from these lines could be obtained more quickly than with conventional crop cultivars (34). Their small size and rapid development make them excellent candidates 
for use as model crops in studies of clubroot under controlled conditions, where space is at a premium. Recently, the clubroot reactions of selected lines of Brassica vegetables, RCBC, and canola to pathotype 6 were assessed under field conditions (1). However, the reaction of RCBC lines to the other common pathotypes of $P$. brassicae is not known. Similarly, A. thaliana has been used for research on clubroot $(2,14,18,20,26)$ but the reaction to the various pathotypes is mostly unknown.

The present study was undertaken to examine the reaction of selected lines of the RCBC and A. thaliana to pathotypes 2, 3, 5, and 6 of $P$. brassicae. Lines with a differential reaction to a pathotype may be useful as model crops for studies of clubroot on canola, vegetables, and other commercial crops. Any differential reaction to pathotypes among the lines might be used to study resistance reactions or, in the future, to develop additional methods to distinguish the pathotypes of $P$. brassicae in Canada.

\section{Materials and Methods}

Plant materials. The Wisconsin Fast Plants or RCBC lines $B$. carinata (L.) A. Braun (genome designation BCbbcc, haploid chromosome number for the $\mathrm{b}$ and $\mathrm{c}$ genomes, $\mathrm{bc}=17)$, B. juncea $(\mathrm{L}$.) Czern (ABaabb, ab = 18), B. napus (L.) (ACaacc, ac = 19), B. oleracea $(\mathrm{Ccc}, \mathrm{c}=9$ ), and $B$. rapa (Aaa, $\mathrm{a}=10$, standard rapid cycling) were obtained from the RCBC, University of Wisconsin, Madison. Shanghai pak choy (B. rapa subsp. Chinensis (Rupr.) var. communis Tsen and Lee) 'Mei Qing Choy' and Chinese flowering cabbage (B. rapa subsp. Chinensis (Rupr.) var. utilis Tsen and Lee) 'Tsoi-sim' were obtained from Stokes Seeds Inc. In total, 84 lines of $A$. thaliana that readily produced viable seed were selected from a set of 96 lines at the NRC Plant Biotechnology Institute, SK, Canada. These stocks, originally obtained from the Arabidopsis Biological Resource Center, Columbus, $\mathrm{OH}$, represent a breadth of geographical origin and genetic diversity $(21,24)$.

Pathogen material. Four pathotypes of $P$. brassicae (pathotypes 2, 3, 5, and 6, William's system) collected from clubbed roots of canola grown in Quebec, central Alberta, southern Alberta, and Ontario, Canada, respectively, were used in this study. They were distinguished based on pathogenicity on the differential set of Williams or using the ECD set $(28,29)$. In addition, one single-sporederived isolate each of pathotypes 3 and 6 (35), from Napa cabbage (B. rapa subsp. Pekinensis (Lour.) Hanlet) 'Granaat', provided by the Strelkov lab, University of Alberta, AB, Canada, were also assessed. The single-spore-derived isolates were increased on the susceptible Shanghai pak choy Mei Qing Choy. Inoculum was prepared from clubbed roots that had been stored at $-20^{\circ} \mathrm{C}$. Spores were extracted by thawing the frozen clubs at room temperature, then homogenizing approximately $3 \mathrm{~g}$ in $100 \mathrm{ml}$ of water at high speed for $2 \mathrm{~min}$ and straining the resulting spore suspension through eight layers of cheesecloth. The spore concentration was determined using a hemocytometer and adjusted to $3 \times 10^{6}$ resting spores $\mathrm{ml}^{-1}$. Freshly prepared inoculum was used for each inoculation.

Cultivation and inoculation of plants. The RCBC lines were grown in tall plastic pots (21-by-3.8-cm Conetainers; Stuewe and Sons Inc.) filled with soil-less mix (Sunshine mix number 4; Sun Gro Horticulture Canada Ltd.). Seedlings were thinned to one per pot after 5 days. Plants were maintained in a growth room at 20 and $25^{\circ} \mathrm{C}$ (day and night, respectively) temperature with $75 \%$ relative humidity and a 16-h photoperiod, watered daily with demineralized water adjusted to $\mathrm{pH} 6.3$ using commercial vinegar $(5 \%$ acetic acid), and fertilized weekly with $20 \mathrm{ml}$ of mineral fertilizer adjusted to $\mathrm{pH} 6.3$ (80 g of 15:15:18 NPK fertilizer per liter stock solution, $5 \mathrm{ml}$ stock solution per liter fertilizer solution; Plant Products Co. Ltd.). Each seedling was inoculated (field-collected isolates only) by pipetting $5 \mathrm{ml}$ of resting spore suspension $(3 \times$ $10^{6}$ spores $/ \mathrm{ml}$ ) at the base of the seedling. The control plants were mock-inoculated with deionized water. In addition, Shanghai pak choy Mei Qing Choy and Chinese flowering cabbage Tsoi-sim (both highly susceptible) were included as controls. The trial was laid out in a randomized complete block design with four replicates (10 plants per rep), and the entire trial was repeated.
The plants were harvested 6 weeks after inoculation. The roots were washed and assessed for clubroot incidence (CI) and severity, based on visual symptoms of root clubbing. Plants were separated into classes using a standard 0 -to- 3 scale, where $0=$ no clubbing, 1 $=$ less than one-third of root clubbed, $2=$ one-third to two-thirds of root clubbed, and $3=$ greater than two-thirds of roots clubbed $(15,35)$. A disease severity index (DSI) was calculated using the following equation (29): DSI $=\{\Sigma[$ (class number)(number of plants in each class)]/[(total number plants per sample)(number of classes -1$)]\} \times 100$.

The resistance response of the lines to $P$. brassicae was classified based on their mean DSI value, as follows: (i) resistant $=0$ to 33 DSI, (ii) intermediate $=34$ to 67 DSI, and (iii) susceptible $=68$ to 100 DSI.

The clubroot reaction of lines of A. thaliana was evaluated in two phases. In the first phase, the 84 lines were evaluated for clubroot reaction using field-collected isolates of pathotypes 2, 3, 5 , and 6 and single-spored isolates of pathotypes 3 and 6 (experiment 1). In the second phase, assessment of the 11 lines that displayed a resistant reaction to one or more pathotypes in experiment 1 was repeated (experiment 2). Each experiment was conducted and assessed using the same methods as for the RCBC lines, except as follows. The trial was laid out in a randomized complete block design with four replicates and eight plants per rep. The seed was stratified at $4^{\circ} \mathrm{C}$ in the dark for a week in damp soil-less mix; then, 14-day-old seedlings were transplanted individually into the cells of 96 -cell plug trays (each cell $=3$ by 2 by $6 \mathrm{~cm}$ ) and inoculated with $3 \mathrm{ml}$ of $3 \times 10^{6}$ spores $/ \mathrm{ml}$ spore suspension. To avoid cross-contamination, the plants in each pathotype treatment (plus the noninoculated control) were grown in separate trays. Due to space and time constraints, only 11 lines of $A$. thaliana could be assessed at a time; therefore, Shanghai pak choy Mei Qing Choy was included in each set of inoculations as an internal control.

Data analysis. Treatment effects were assessed using the mixedmodel analysis of variance of the data with the lines or species as the fixed effect and replication as a random effect (PROC MIXED, SAS software version 9.2; SAS Institute Inc.). The data set for each trial was tested for normality using the Shapiro-Wilk test of residuals, and checked for outliers using Lund's test of standardized residuals (19). No outliers were found in any data set. For the $\mathrm{RCBC}$ trial, there was no effect of repetition or repetitiontreatment; therefore, the data were pooled across repetition for analysis. Means were separated using Tukey's test. The relationship between growth room assessments of the RCBC lines and previous assessments under field conditions (1) was examined using Pearson's correlation coefficient. For the assessment of Arabidopsis lines, the consistency of response in the 11 selected lines between the two repetitions was examined using Pearson's correlation coefficient. The relationship between the field pathotypes and single-spore isolates on the 84 Arabidopsis lines was also examined using Pearson's correlation coefficient.

Prior to analysis, percent data were arcsine-transformed when necessary to improve the normality and homogeneity of variance but nontransformed means are presented for uniformity of presentation. Differences were significant at $P \leq 0.05$ unless otherwise noted.

\section{Results}

In both repetitions of the assessment of RCBC lines, CI and DSI were $100 \%$ in the inoculated controls (Mei Qing Choy and Tsoisim) and no symptoms of clubroot developed in any noninoculated control (data not shown). Therefore, all of the control treatments were excluded from subsequent analyses. Also, there was no effect of repetition or repetition-treatment; therefore, the data from the two repetitions of the trial were combined for analysis. CI and DSI showed a similar pattern of response to treatment (Fig. 1); therefore, the focus in this presentation will be on DSI. The mean DSI of the pathotypes, listed in decreasing order, was pathotype 6 (DSI $=61 \%)$, pathotype $3(32 \%)$, pathotype $5(26 \%)$, and pathotype 2 $(12 \%)$, with a standard error (SE) of $4.3 \%$. The mean DSI for the 
RBCB lines ( $\mathrm{SE}=4.1 \%$ ), listed in decreasing order of susceptibility, was $B$. juncea $(\mathrm{DSI}=59 \%)$, B. carinata $(34 \%)$, B. rapa $(24 \%)$, $B$. napus $(12 \%)$, and $B$. oleracea $(9 \%)$. However, because there was a line-pathotype interaction, the mean reaction (main effects) does not always reflect the important relationships, and reactions of single lines to individual pathotypes were investigated in more detail.

Some of the RCBC lines showed a differential response to pathotype (Fig. 1; Table 1). Four of five RCBC lines were resistant to pathotypes 2 and 5 . The exception was B. juncea, which had an intermediate response to pathotype $2(\mathrm{DSI}=55 \%)$ and was sus- ceptible to pathotype 5 (68\%). Similarly, two of five RCBC lines were resistant to pathotype 3 while $B$. carinata, $B$. rapa, and $B$. napus had an intermediate response (Fig. 1). B. carinata $(91 \%)$ and $B$. juncea $(100 \%)$ were highly susceptible to pathotype $6, B$. oleracea $(40 \%)$ and B. rapa $(66 \%)$ had an intermediate response, and B. napus was resistant (2\%) (Fig. 1).

There was a strong positive correlation $(r=0.91, P<0.0001)$ when the DSI values of RCBC lines inoculated with pathotype 6 under controlled conditions in the current study were compared with results from a previous field trial conducted over several years at a site where pathotype 6 was dominant (1).
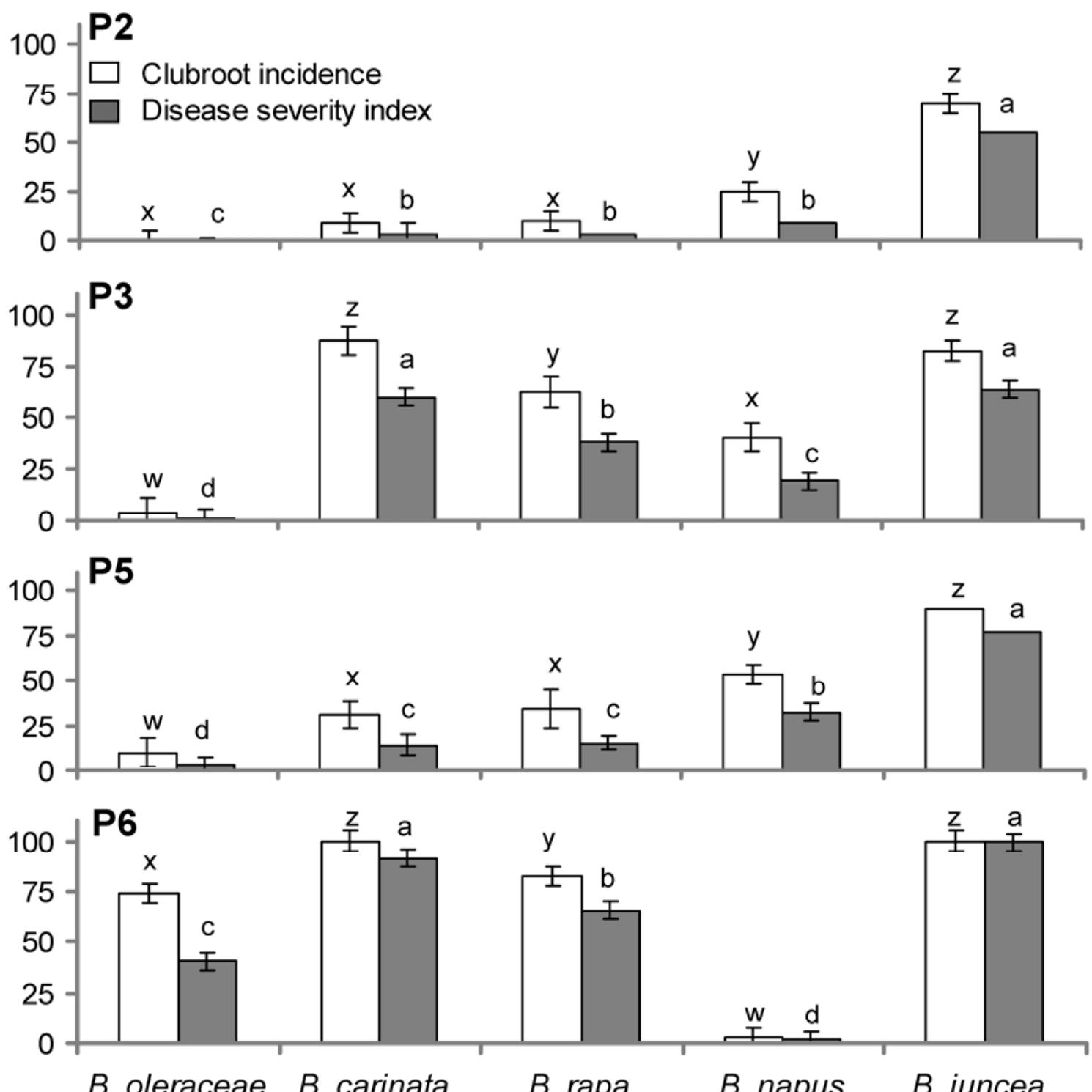

Fig. 1. Clubroot incidence (\%) and severity (disease severity index) of the Rapid Cycling Brassica Collection lines inoculated with field-collected Plasmodiophora brassicae pathotypes 2, 3, 5 and 6 . Bars with the same letter above do not differ based on Tukey's test at $P<0.05$. Capped lines represent \pm standard error.

Table 1. Analysis of variance of clubroot incidence (CI) and disease severity index (DSI) for the Rapid Cycling Brassica Collection lines inoculated with field-collected isolates of Plasmodiophora brassicae (data combined across two repetitions of the trial, each with four replicates)

\begin{tabular}{|c|c|c|c|c|c|c|c|}
\hline \multirow[b]{2}{*}{ Source } & \multirow[b]{2}{*}{ df } & \multicolumn{3}{|c|}{ CI } & \multicolumn{3}{|c|}{ DSI } \\
\hline & & Mean square & $F$ value & $P$ value & Mean square & $F$ value & $P$ value \\
\hline Replication & 7 & 132 & 2 & 0.21 & 57 & 1.7 & 0.12 \\
\hline Pathotype & 3 & 17,303 & 307 & 0.0001 & 14,612 & 432 & 0.0001 \\
\hline Line & 4 & 19,758 & 350 & 0.0001 & 20,027 & 592 & 0.0001 \\
\hline Pathotype $\times$ line & 12 & 5,155 & 91 & 0.0001 & 3,113 & 92 & 0.0001 \\
\hline $\mathrm{CV}(\%)^{\mathrm{a}}$ & $\ldots$ & $\ldots$ & 16 & $\ldots$ & $\ldots$ & 17 & $\ldots$ \\
\hline
\end{tabular}

${ }^{a}$ Coefficient of variation. 
In experiment 1 of the assessment of A. thaliana, DSI in Shanghai pak choy Mei Qing Choy was $100 \%$ for each of the pathotypes in each run (data not shown). Based on this uniform response in the susceptible control, the data from the individual runs were pooled for analysis. Overall, pathotype 3 (mean DSI $=88 \%$ ) and pathotype $6(86 \%)$ produced the most severe symptoms, pathotype 5 was intermediate $(79 \%)$, and pathotype $2(69 \%)$ produced the least severe symptoms on the 84 lines assessed $(\mathrm{SE}=0.46 \%)$. As for the RCBC lines, a strong line-pathotype interaction was found (Supplementary Table S1) and individual combinations were examined.

None of the Arabidopsis lines was resistant to all of the pathotypes, and the susceptible lines had various forms of galls compared with the resistant and noninoculated control lines (Fig. 2). Lines Ct-1(mean DSI $=0 \%)$, Hr-10 (15\%), Mrk-0 (18\%), Bay-0 (20\%), Ler-1(20\%), Col-0 (22\%), Pro-0 (22\%), Ed-1 (24\%), Uod-1 (25\%), Wa-1 (26\%), Wt-0 (29\%), and Uod-7 (31\%) were resistant to pathotype 2; lines Pu2-23 (8\%), Knox-10 (23\%), C24 (25\%), Uod-1 (26\%), and Nok-3 (29\%) were resistant to pathotype 5; and lines Sorbo (6\%), Ws-2 (8\%), Ct-1(18\%), O-27(20\%), and NFA-8 (22\%) were resistant to pathotype 6 . Only two lines, Nok-3 $(29 \%)$ and Kz-9 (32\%), were resistant to pathotype 3. Each of the other lines had a susceptible or intermediate reaction to all of the pathotypes.

In experiment 2 , there was a strong line-pathotype interaction for both CI and DSI in analysis of variance. Overall, pathotype 3 produced the most severe symptoms (mean DSI $=92 \%$ ), pathotype $5(67 \%)$ and pathotype $6(61 \%)$ were intermediate, and pathotype 2 produced the fewest symptoms $(56 \%)(\mathrm{SE}=0.23 \%)$. Lines Ct-0 $(\mathrm{DSI}=0 \%), \mathrm{Hr}-10(15 \%)$, Ler-1(17\%), and Col-0 (18\%) were resistant to pathotype $2(\mathrm{SE}=1.4 \%)$; Nok-3 $(20 \%)$ was resistant to pathotype $3(\mathrm{SE}=1.9 \%)$; Pu2-23 $(6 \%)$ and Nok-3 (31\%) were resistant to pathotype $5(\mathrm{SE}=2.6 \%)$; and Ws-2 $(5 \%)$, Sorbo $(6 \%)$, Ct-0 (16\%), and NFA-8 (22\%) were resistant to pathotype $6(\mathrm{SE}=$ $1.9 \%)$.

The pattern of response to pathotype of the 11 selected lines in experiments 1 and 2 was highly correlated ( $r=0.98$ for CI, $r=$ 0.96 for DSI); therefore, the mean response for each line to pathotype across the two experiments is presented (Fig. 3). In contrast, the reaction of several of the 84 Arabidopsis lines to the singlespore isolates differed substantially from that of the field collections of pathotypes 3 ( $r=0.08$ for CI, $r=0.07$ for DSI) and $6(r=$ 0.09 for CI, $r=0.09$ for DSI) (Fig. 4). There was a strong linepathotype interaction for both CI and DSI in analysis of variance
(Table 2). Overall, the field collections produced slightly more symptoms than the single-spore isolates (mean $\mathrm{CI}=96$ versus $90 \%$, DSI $=88$ versus $83 \%$ for pathotype $3 ; \mathrm{CI}=92$ versus $83 \%$, $\mathrm{DSI}=86$ versus $73 \%$ for pathotype $6 ; \mathrm{SE}=0.35 \%$ for $\mathrm{CI}, 0.47 \%$ for DSI). Of the 84 Arabidopsis lines, 62 lines had the same DSI reaction (susceptible, intermediate, and resistant) to the singlespore and field collection isolates of pathotype 3, and 46 lines had the same response to isolates of pathotype 6 (Fig. 4). For example, Hr-5, Hr-10, Kin-0, and Van-0 were susceptible to the field collection and resistant to single-spore isolates of pathotype 3 (Fig. 4).

\section{Discussion}

The RBCB lines in this study showed a differential reaction to the four pathotypes of $P$. brassicae that were assessed: $B$. napus was resistant to each of the pathotypes; $B$. oleracea was resistant to pathotypes 2,3 , and $5 ; B$. carinata and $B$. rapa were resistant to pathotypes 2 and 5 ; and $B$. juncea had an intermediate response to pathotypes 2 and 3 . This indicates that these RCBC lines could be useful as model crops for many types of research on clubroot. The advantage of RCBC lines as model crops is that a line can be chosen that is similar in growth habit and root and shoot architecture to the crop of interest. Also, these lines are small in stature and set seed within 42 days; therefore, they are suitable for use where space is at a premium (e.g., controlled conditions). Finally, although seed of the RCBC lines is expensive relative to Shanghai pak choy and canola, the lines have been continually available for many years and, therefore, are likely to be consistently available over time. This represents a substantial advantage over commercial canola cultivars that can be removed from the marketplace without notice (1)

Most of the $84 \mathrm{~A}$. thaliana lines were susceptible to each pathotype of $P$. brassicae and no line was resistant to all four pathotypes. Lines that did show a differential reaction to pathotype were generally moderately resistant, and none were immune. However, 11 of the 84 lines displayed a resistant reaction (DSI > 33\%) to at least one pathotype, and the reaction of these 11 lines was highly consistent between experiments 1 and 2 ( $r=0.98$ for $\mathrm{CI}, r=0.96$ for DSI). This consistent response indicates the reproducibility of the experiment and the accuracy of the results. The sequenced genome and the availability of mutants make Arabidopsis lines very useful for molecular studies. However, the seed is very small and must be vernalized to ensure adequate germination, and the plant architecture is not similar to most Brassica crops; therefore, Arabidopsis has some drawbacks as a model crop for many other purposes.
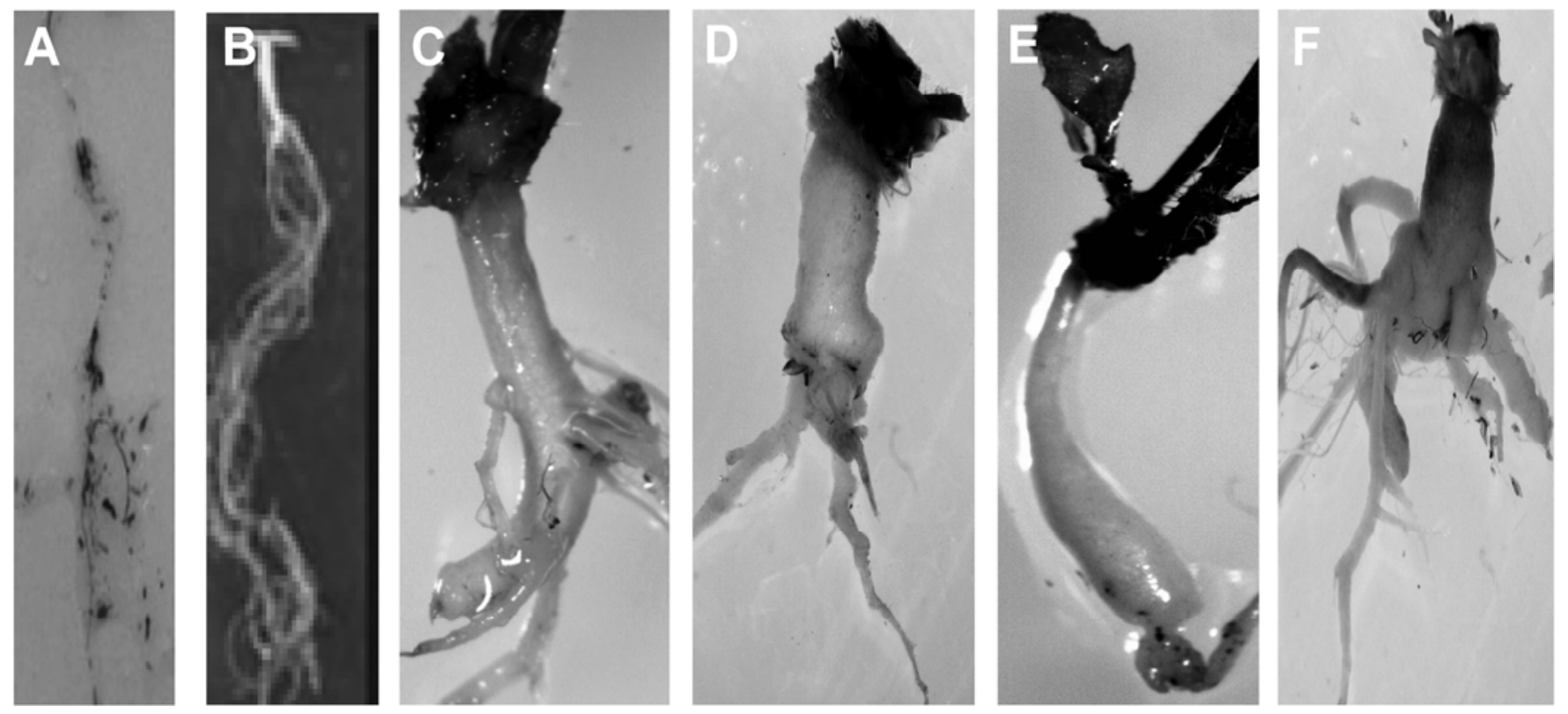

Fig. 2. Roots of A, the noninoculated (control); B, inoculated resistant line; and C-F, range of symptom development in Arabidopsis lines. 
In previous studies, pathotype 3 consistently produced more severe symptoms of clubroot than pathotype $6(10,29)$. Also, canola genotypes with resistance to pathotype 3 or 5 were generally also resistant to other pathotypes (29). In the current trial, pathotypes 3 and 6 produced similar levels of clubroot on the 84 lines of $A$. thaliana but pathotype 6 produced more severe symptoms than pathotype 3 when averaged over the five RCBC lines. Also, the lines that were resistant to pathotype 3 were not necessarily resistant to pathotypes 2, 5, or 6 (Table 3; Figs. 1 and 3).

There was a strong correlation between clubroot severity on RCBC lines grown at a field site where pathotype 6 was dominant (1) and in the controlled environment trials with pathotype 6 . This indicates that the pathogen population was uniform and stable. In contrast, the single-spored isolates of pathotypes 3 and 6 did not produce the same pattern of resistance responses as the field collections on several of the A. thaliana lines. This supports previous reports of heterogeneity of the pathogen in field collections $(4,16,17,25,35)$. One pathotype may be dominant and easily identified, whereas others may be present at a low frequency and, thus, easily overlooked. However, components of a population that are present at low frequency can be of great interest, especially if they are virulent on cultivars that are resistant to the predominant pathotype (16). The use of specific pathotypes of $P$. brassicae with defined virulence factors, alone and in mixtures, has been recommended to improve the efficiency of selection in resistance breeding (16). Additional research is required to determine the

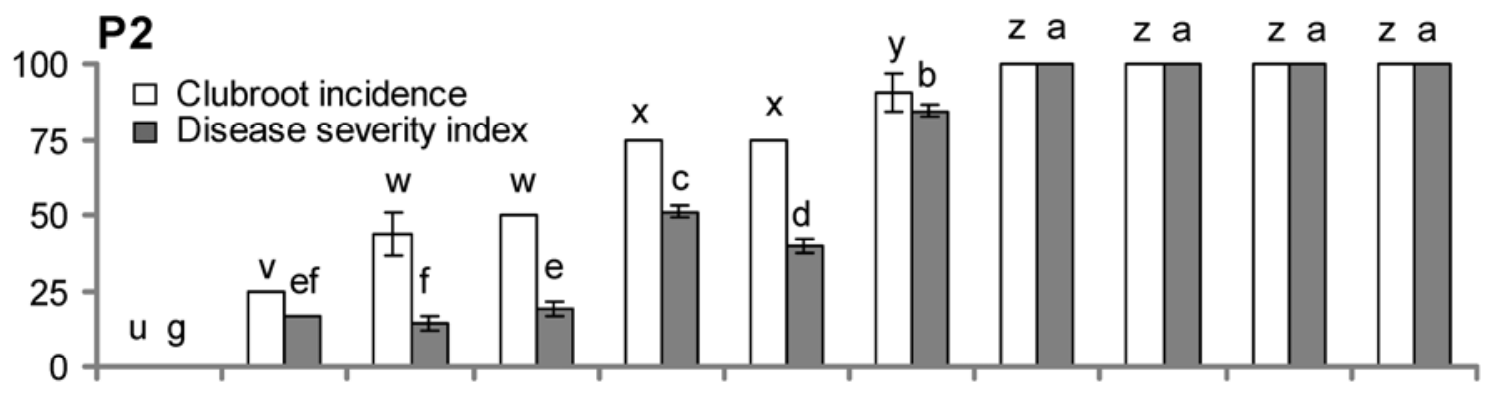

P3
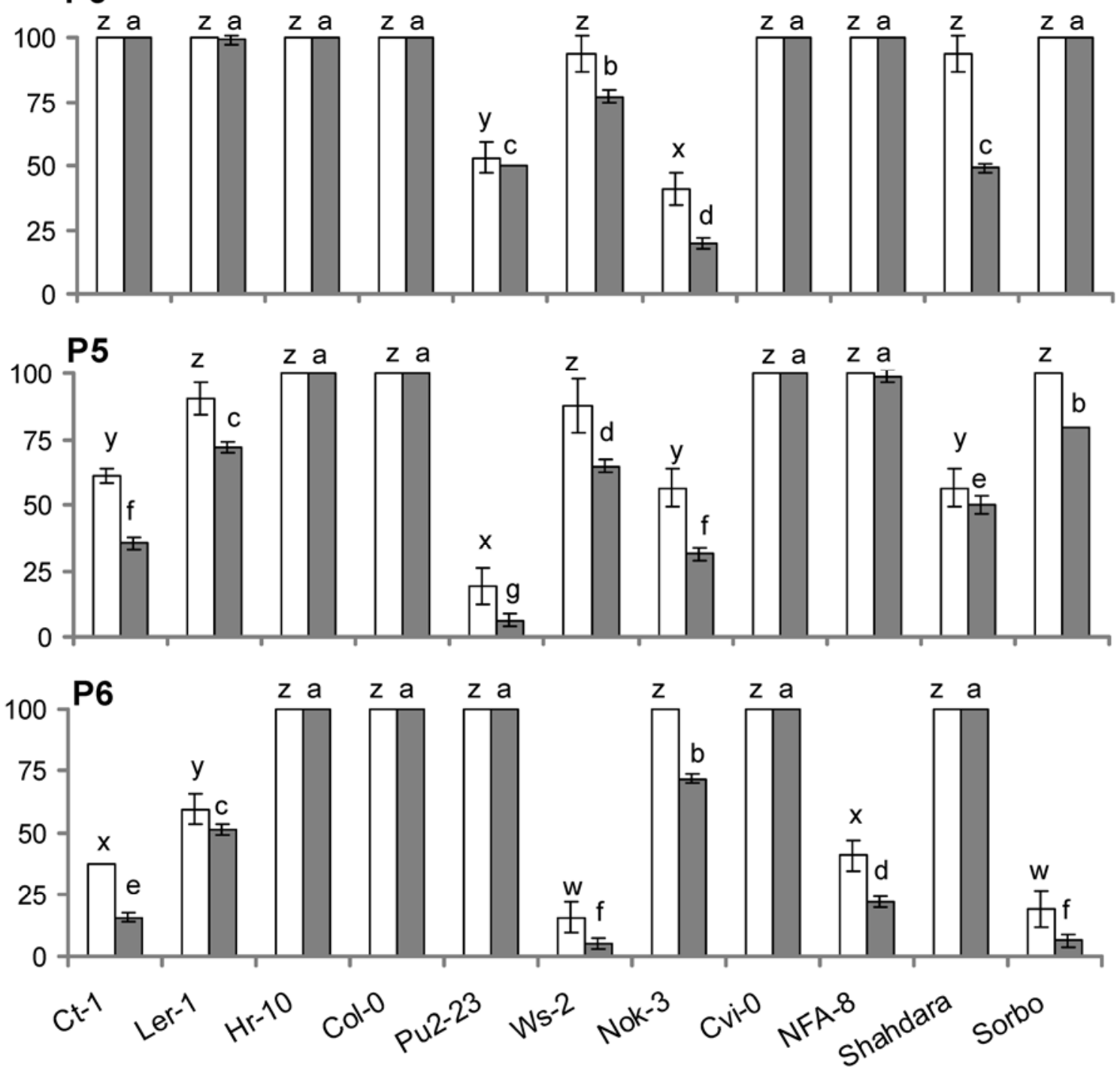

Fig. 3. Mean clubroot incidence and severity of 11 Arabidopsis lines to field-collected Plasmodiophora brassicae pathotypes 2, 3, 5, and 6 in experiments 1 and 2. Bars with the same letter above do not differ based on Tukey's test at $P<0.05$. Capped lines represent \pm standard error. 
reaction pattern of a selected host against a wider range of singlespored isolates.

Inclusion of the susceptible Shanghai pak choy Mei Qing Choy and Chinese cabbage Tsoi-sim in the RCBC trial and Mei Qing Choy in the Arabidopsis experiments was very useful. Several sets of inoculations (repetitions in the RCBC experiment, subsets of Arabidopsis lines in experiment 1) could be combined with confidence because of the high and consistent levels of clubroot in each inoculated control treatment. Both crops were highly susceptible to each of the P. brassicae pathotypes. Previous studies had shown that these cultivars were highly susceptible to pathotype $6(1,10)$ but this is the first time that the reaction of these potential model crops has been assessed against a wider range of pathotypes. Seed for Shanghai pak choy Mei Qing Choy and Chinese cabbage Tsoisim is readily available commercially and inexpensive; the seed germinates easily, seedlings are vigorous, and the plants grow quickly but are small in stature. We conclude that both Mei Qing Choy and Tsoi-sim have potential for use as susceptible checks in future clubroot studies.
There have been a number of suggestions for how to classify host reaction to $P$. brassicae, based on various cut-off points for DSI $(5,8,15,29,31)$. The cut-off points for DSI include $20 \%$ (31), $33 \%(5,8)$, and $49 \%$ (29). Clubroot reaction in the current study was separated into three categories: (i) resistant $=0$ to $33 \%$ DSI, (ii) intermediate $=34$ to $67 \%$ DSI, and (iii) susceptible $=68$ to $100 \%$ DSI. Although the focus of the current work was on substantial and consistent differences in reaction (resistant versus susceptible) to differentiated pathotypes, the intermediate category of reaction may represent a fruitful area for researchers interested in quantitative resistance and factors that influence the expression of resistance.

Several sets of differential hosts have been proposed and employed in recent years $(4,5,15,27,29,33,35)$. Unfortunately, comparison and identification of the pathotypes described in these different systems is not always straightforward. Substantial variability in the reaction of $P$. brassicae has been observed on the differential lines in each of the three most commonly utilized sets $(5,27,35)$. Also, there is a concern that certain of these differentials
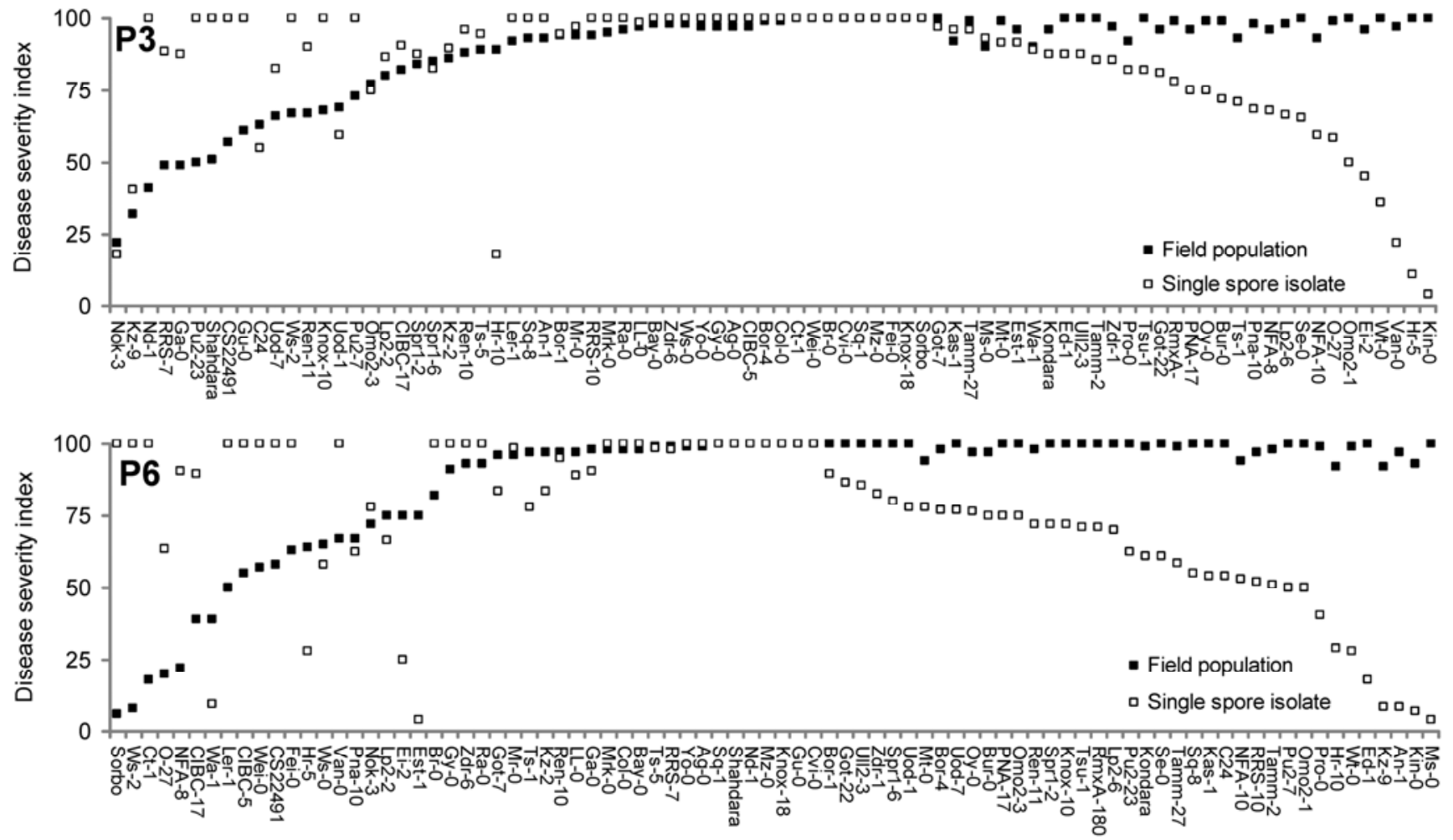

Fig. 4. Clubroot severity (disease severity index) of 84 Arabidopsis lines inoculated with a field collected or single-spore isolate of pathotypes A, 3 and B, 6 of Plasmodiophora brassicae.

Table 2. Analysis of variance of clubroot incidence (CI) and disease severity index (DSI) for 84 lines of Arabidopsis thaliana inoculated with a fieldcollected or single-spored isolate of pathotypes 3 and 6 of Plasmodiophora brassicae

\begin{tabular}{|c|c|c|c|c|c|c|c|}
\hline \multirow[b]{2}{*}{ Source $^{a}$} & \multirow[b]{2}{*}{ df } & \multicolumn{3}{|c|}{ CI } & \multicolumn{3}{|c|}{ DSI } \\
\hline & & Mean square & $F$ value & $P$ value & Mean square & $F$ value & $P$ value \\
\hline \multicolumn{8}{|l|}{ Pathotype 3} \\
\hline Replication & 3 & 39 & 1.4 & 0.24 & 89 & 1.5 & 0.23 \\
\hline Isolate & 1 & 4,934 & 180 & 0.0001 & 2,108 & 35 & 0.0001 \\
\hline Line & 83 & 1,049 & 39 & 0.0001 & 1,641 & 27 & 0.0001 \\
\hline Isolate $\times$ line & 83 & 799 & 29 & 0.0001 & 1,362 & 22 & 0.0001 \\
\hline $\mathrm{CV}(\%)$ & & & 6 & & & 9 & \\
\hline \multicolumn{8}{|l|}{ Pathotype 6} \\
\hline Replication & 3 & 24 & 0.5 & 0.7 & 12 & 0.2 & 0.91 \\
\hline Isolate & 1 & 12,365 & 247 & 0.0001 & 26,176 & 387 & 0.0001 \\
\hline Line & 83 & 1,719 & 38 & 0.0001 & 2,288 & 34 & 0.0001 \\
\hline Isolate $\times$ line & 83 & 1,852 & 41 & 0.0001 & 2,574 & 38 & 0.0001 \\
\hline CV (\%) & $\ldots$ & $\ldots$ & 8 & $\ldots$ & $\ldots$ & 10 & $\ldots$ \\
\hline
\end{tabular}

${ }^{a}$ Field versus single-spore isolate of each pathotype; $\mathrm{CV}=$ coefficient of variation. 
Table 3. Lines of Arabidopsis, Rapid Cycling Brassica Collection (RCBC), and Shanghai pak choy that could be used to differentiate the predominant pathotypes of Plasmodiophora brassicae in Canada

\begin{tabular}{llllll}
\hline & & \multicolumn{4}{c}{ Pathotype $^{\mathbf{a}}$} \\
\cline { 3 - 6 } Plant species & Line & $\mathbf{2}$ & $\mathbf{3}$ & $\mathbf{5}$ & $\mathbf{6}$ \\
\hline Arabidopsis thaliana & Sorbo & $\mathrm{S}$ & $\mathrm{S}$ & $\mathrm{S}$ & $\mathrm{R}$ \\
& Ct-1 & $\mathrm{R}$ & $\mathrm{S}$ & $\mathrm{I}$ & $\mathrm{R}$ \\
Brassica carinata & RCBC & $\mathrm{R}$ & $\mathrm{I}$ & $\mathrm{R}$ & $\mathrm{S}$ \\
B. napus & RCBC & $\mathrm{R}$ & $\mathrm{R}$ & $\mathrm{R}$ & $\mathrm{R}$ \\
B. rapa subsp. chinensis & Pak choy & $\mathrm{S}$ & $\mathrm{S}$ & $\mathrm{S}$ & $\mathrm{S}$ \\
\hline
\end{tabular}

a Pathotype designations correspond to Williams (33). The reaction of RCBC and Arabidopsis lines to P. brassicae was classified into three categories based on disease severity index value: (i) $\mathrm{R}$ (resistant) $=0$ to $33 \%$ DSI, (ii) I (intermediate) $=34$ to $67 \%$ DSI, and (iii) $\mathrm{S}$ (susceptible) $=$ 68 to $100 \%$ DSI.

lack genetic homogeneity $(8,27)$. This likely indicates that these differentials do not reflect the complete range of pathogenic diversity in pathogen populations. An improved differential series in which each of the hosts is genetically uniform would be very useful. Also, these differentials were originally developed to study populations of $P$. brassicae on Brassica vegetables and, therefore, may not cover the range of pathogen diversity on canola. This variability has been mentioned as a concern in the Northern Great Plains, where clubroot is an emerging problem on canola $(12,13)$.

Seed of lines chosen for any differential set should be readily and consistently available. There have been problems obtaining seed of some of the lines of the ECD differential set $(27,29)$, especially the seed of the susceptible species in the ECD series, Napa cabbage (B. rapa subsp. Pekinensis (Lour.) Hanlet) Granaat. It is also helpful if the plants are easy to grow and handle. Scott et al. (25) recommended that the hosts should ideally be commercial cultivars in current use. This approach might be suitable for certain vegetable crops, where some cultivars can remain in production for decades, but would not be practical for canola because cultivars go out of production quickly.

It will take extensive testing to develop a new differential set of hosts for $P$. brassicae pathotypes but the differential response of RCBC and Arabidopsis lines might be useful in this process. For example, the lines of Arabidopsis and RCBC in Table 3 could be used to identify the four main pathotypes of $P$. brassicae examined in this study. The RBCB line of B. napus was resistant and the Shanghai pak choy Mei Qing Choy was susceptible to all the pathotypes. RCBC line B. carinata was resistant to pathotype 5 and intermediate to pathotype 3 . This is a weaker line in the differential set, and it would be useful to identify a line that had a stronger differential reaction to these two pathotypes. Line Ct-1 was resistant to pathotype 2 but susceptible to pathotype 3 , while Sorbo was susceptible to both pathotypes; $B$. carinata was resistant to pathotype 5, while Sorbo was susceptible; and B. carinata was susceptible to pathotype 6 but Sorbo and Ct-1 were resistant.

The current study examined the reaction of lines of the RCBC and A. thaliana to the predominant pathotypes of $P$. brassicae in Canada. We conclude that many of the RCBC and Arabidopsis lines in this study can serve as model plants for research on clubroot, including Mei Qing Choy, which is highly susceptible to the four pathotypes. The RCBC lines, in particular, are readily available and easy to grow. The reaction between the single-spored isolates of pathotypes 3 and 6 differed with reaction to the field collections on several of the 84 Arabidopsis lines and warrants further study. Development of a differential system for the pathotypes of $P$. brassicae in the Northern Great Plains may be of value in breeding for resistance and management of clubroot in canola, and the lines identified in this study may have a role in a new system.

\section{Acknowledgments}

We thank P. Ashe, Plant Biotechnology Institute, National Research Council, SK, Canada, for providing seed of the Arabidopsis lines for assessment; the Clubroot Risk Mitigation Initiative of Agriculture and Agri-Food Canada for partial funding of the project; and X. Junzhong, visiting fellow at the Department of Plant Agriculture, University of Guelph, for technical assistance.

\section{Literature Cited}

1. Adhikari K. K. C., Gossen, B. D., and McDonald M. R. 2012. Reaction to Plasmodiophora brassicae pathotype 6 in lines of Brassica vegetables, Wisconsin Fast Plants, and canola. HortScience 47:374-377.

2. Agarwal, A., Kaul, V., Faggian, R., and Cahill, D. M. 2009. Development and use of a model system to monitor clubroot disease progression with an Australian field population of Plasmodiophora brassicae. Aust. Plant Pathol. 38:120-127.

3. Ayers, G. W. 1957. Races of Plasmodiophora brassicae. Can. J. Bot. 35:923-932.

4. Ayers, G. W., and Lelacheur, K. E. 1972. Genetics of resistance in rutabaga to two races of Plasmodiophora brassicae. Can. J. Plant Sci. 52:897-900.

5. Buczacki, S. T., Toxopeus, H., Mattusch, P., Johnston, T. D., Dixon, G. R., and Hobolth, L. A. 1975. Study of physiologic specialization in Plasmodiophora brassicae: proposals for attempted rationalization through an international approach. Trans. Br. Mycol. Soc. 65:295-303.

6. Cao, T., Manolii, V. P., Hwang, S. F., Howard, R. J., and Strelkov, S. E. 2009. Virulence and spread of Plasmodiophora brassicae [clubroot] in Alberta, Canada. Can. J. Plant Pathol. 31:321-329.

7. Dixon, G. R. 2009. Plasmodiophora brassicae in its environment. J. Plant Growth Regul. 28:212-228.

8. Dobson, R. L., Robak, J., and Gabrielson, R. L. 1983. Pathotypes of Plasmodiophora brassicae in Washington, Oregon, and California. Plant Dis. 67:269-271.

9. Donald, E. C., Cross, S. J., Lawrence, J. M., and Porter, I. J. 2006. Pathotypes of Plasmodiophora brassicae, the cause of clubroot, in Australia. Ann. Appl. Biol. 148:239-244.

10. Gossen, B. D., Adhikari, K. K. C., and McDonald, M. R. 2012. Effects of temperature on infection and subsequent development of clubroot under controlled conditions. Plant Pathol. 61:593-599.

11. Hildebrand, P. D., and McRae, K. B. 1998. Control of clubroot caused by Plasmodiophora brassicae with non-ionic surfactants. Can. J. Plant Pathol. 20:1-11.

12. Howard, R. J., Strelkov, S. E., and Harding, M. W. 2010. Clubroot of cruciferous crops-new perspective on an old disease. Can. J. Plant Pathol. 32:43-57

13. Hwang, S. F., Strelkov, S. E., Feng, J., Gossen, B. D., and Howard, R. J. 2012. Plasmodiophora brassicae: a review of an emerging pathogen of the Canadian canola (Brassica napus) crop. Mol. Plant Pathol. 13:105-113.

14. Kobelt, P., Siemens, J., and Sacristan, M. D. 2000. Histological characterization of the incompatible interaction between Arabidopsis thaliana and the obligate biotrophic pathogen Plasmodiophora brassicae. Mycol. Res. $2: 220-225$

15. Kuginuki, Y., Yoshikawa, H., and Hirai, M. 1999. Variation in virulence of Plasmodiophora brassicae in Japan tested with clubroot-resistant cultivars of Chinese cabbage (Brassica rapa L. ssp. Pekinensis). Eur. J. Plant Pathol. 105:327-332.

16. Jones, D. R., Ingram, D. S., and Dixon, G. R. 1982. Characterization of isolates derived from single resting spores of Plasmodiophora brassicae and studies of their interaction. Plant Pathol. 31:239-246.

17. Lammerink, J. 1965. Six pathogenic races of Plasmodiophora brassicae Wor. in New Zealand. N. Z. J. Agric. Res. 8:156-164.

18. Ludwig-Müller, J. 2009. Glucosinolates and the clubroot disease: defense compounds or auxin precursors? Phytochem. Rev. 8:135-148.

19. Lund, R. E. 1975. Tables for an approximate test for outliers in linear models. Techometrics 17:473-476.

20. Mithen, R., and Magrath, R. 1992. A contribution to the life history of Plasmodiophora brassicae: secondary plasmodia development in root galls of Arabidopsis thaliana. Mycol. Res. 96:877-885.

21. Nordborg, M., Hu, T. T., Ishino, Y., Jhaveri, J., Toomajian, C., Zheng, H., Bakker, E., Calabrese, P., Gladstone, J., Goyal, R., Jakobsson, M., Kim, S., Morozov, Y., Padhukasahasram, B., Plagnol, V., Rosenberg, N. A., Shah, C., Wall, J. D., Wang, J., Zhao, K., Kalbfleisch, T., Schulz, V., Kreitman, M., and Bergelson, J. 2005. The pattern of polymorphism in Arabidopsis thaliana. PLoS Biol. 3:e196.

22. Reyes, A. A., Davidson, T. R., and Marks, C. F. 1974. Races, pathogenicity and chemical control of Plasmodiophora brassicae in Ontario. Phytopathology 64:173-177.

23. Rowe, R. C. 1983. Evaluation of Radish cultivars for resistance to Clubroot (Plasmodiophora brassicae) race 6 for Midwestern United States. Plant Dis. 64:462-464

24. Schmitz, R. J., and Ecker, J. R. 2012. Epigenetic and epigenomic variation in Arabidopsis thaliana. Trends Plant Sci. 17:149-154.

25. Scott, E. S. 1985. Production and characterization of single-spore isolates of Plasmodiophora brassicae. Plant Pathol. 34:287-292.

26. Siemens, J., Graf, H., Bulman, S., In, O., and Ludwig-Müller, J. 2009. Monitoring expression of selected Plasmodiophora brassicae genes during clubroot development in Arabidopsis thaliana. Plant Pathol. 58:130-136.

27. Somé, A., Manzanares, M. J., Laurens, F., Baron, F., Thomas, G., and Rouxel, F. 1996. Variation for virulence on Brassica napus L. amongst Plasmodiophora brassicae collections from France and derived single-spore 
isolates. Plant Pathol. 45:432-439.

28. Strelkov, S. E., Manolii, V. P., Cao, T., Xue, S., and Hwang, S. F. 2007. Pathotype classification of Plasmodiophora brassicae and its occurrence in Brassica napus in Alberta, Canada. J. Phytopathol. 155:706-712.

29. Strelkov, S. E., Tewari, J. P., Smith, E., and Smith-Degenhardt, E. 2006. Characterization of Plasmodiophora brassicae populations from Alberta, Canada. Can. J. Plant Pathol. 28:467-474.

30. Tewari, J. P., Strelkov, S. E., Orchard, D., Hartman, M., Lange, R. M., and Turkington, T. K. 2005. Identification of clubroot of crucifers on canola (Brassica napus) in Alberta. Can. J. Plant Pathol. 27:143-144.

31. Toxopeus, H., Dixon, G. R., and Mattusch, P. 1986. Physiologic specialization in Plasmodiophora brassicae: an analysis by international experi- mentation. Trans. Br. Mycol. Soc. 87:279-286

32. Wallenhammar, A. C. 1996. Prevalence of Plasmodiophora brassicae in a spring oilseed rape growing area in central Sweden and factors influencing soil infestation levels. Plant Pathol. 45:710-719.

33. Williams, P. H. 1966. A system for the determination of races of Plasmodiophora brassicae that infect cabbage and rutabaga. Phytopathology 56:624626.

34. Williams, P. H., and Hill, C. B. 1986. Rapid-cycling populations of Brassica. Sci. New Ser. 232:1385-1389.

35. Xue, S., Cao, T., Howard, R. J., Hwang, S. F., and Strelkov, S. E. 2008 Isolation and variation in virulence of single-spore isolates of Plasmodiophora brassicae from Canada. Plant Dis. 92:456-462. 